\title{
YC-1 reduces inflammatory responses by inhibiting nuclear factor-kB translocation in mice subjected to transient focal cerebral ischemia
}

\author{
WEI-TING LEE ${ }^{1,2}$, SHIH-HUANG TAI ${ }^{1,2}$, YU-WEN LIN ${ }^{2}$, TIAN-SHUNG WU ${ }^{1,3}$ and E-JIAN LEE ${ }^{2}$ \\ ${ }^{1}$ Institute of Biotechnology and Clinical Medicine, National Cheng Kung University, Tainan 70101; \\ ${ }^{2}$ Neurophysiology Laboratory, Neurosurgical Service, Department of Surgery, National Cheng Kung University \\ Medical Center and Medical School, Tainan 70428; ${ }^{3}$ School of Pharmacy, National Cheng Kung University Hospital, \\ College of Medicine, National Cheng Kung University, Tainan 70101, Taiwan, R.O.C.
}

Received March 11, 2016; Accepted July 12, 2017

DOI: $10.3892 / \mathrm{mmr} .2018 .9178$

\begin{abstract}
. 3-(5-hydroxymethyl-2-furyl)-1-benzyl-indazole (YC-1) is understood to protect against ischemic stroke, but the molecular basis for its neuroprotection remains to be fully characterized. The present study investigated the influence of YC-1 on inflammatory responses following experimental stroke. Previous studies indicated that nuclear factor $(\mathrm{NF})-\kappa \mathrm{B}$-driven signals serve a pivotal role in mediating inflammatory responses following stroke. Ischemic stroke results in activation of $\mathrm{NF}-\kappa \mathrm{B}$ to induce gene expression of factors including inducible nitric oxide synthase, interleukin (IL)-1 $\beta$, IL-6 and matrix metalloproteinases (MMPs). The results of the present study demonstrated that YC-1 effectively reduced brain infarction and brain edema, and improved blood-brain barrier leakage. Additionally, animals treated with YC-1 exhibited significant reductions in neutrophil and macrophage infiltration into the ischemic brain. Furthermore, YC-1 effectively inhibited NF- $\kappa \mathrm{B}$ translocation and binding activity, and the activity and expression of MMP-9 following ischemic stroke. In conclusion, YC-1 may effectively attenuate $\mathrm{NF}-\kappa \mathrm{B}$-induced inflammatory damage following cerebral ischemia-reperfusion.
\end{abstract}

Correspondence to: Professor E-Jian Lee, Neurophysiology Laboratory, Neurosurgical Service, Department of Surgery, National Cheng Kung University Medical Center and Medical School, 138 Sheng-Li Road, Tainan 70428, Taiwan, R.O.C.

E-mail: ejian@mail.ncku.edu.tw

Professor Tian-Shung Wu, School of Pharmacy, National Cheng Kung University Hospital, College of Medicine, National Cheng Kung University, 1 University Road, Tainan 70101, Taiwan, R.O.C.

E-mail: tswu@mail.ncku.edu.tw

Key words: YC-1, nuclear factor- $\textrm{B}$, inflammatory, matrix metalloproteinase 9 , ischemic stroke

\section{Introduction}

Ischemic stroke resulting in neurological deficits is the leading cause of acquired disability in adults. Current treatments for acute ischemic stroke are confined to thrombolysis, including tissue plasminogen activator (tPA) and supportive treatment. However, only a small proportion $(\sim 2 \%)$ of stroke patients benefit from tPA treatment (1). Therefore, novel thrombolytic agents or neuroprotective agents are urgently required.

Although various underlying mechanisms are involved in the pathogenesis of stroke, there is increasing evidence demonstrating that inflammation accounts for its progression (2). Following focal cerebral ischemia, resting microglia cells become activated and produce diverse but intense proinflammatory mediators, including nitric oxide (NO), reactive oxygen species (ROS), tumor necrosis factor- $\alpha$ (TNF- $\alpha$ ) and interleukin-1 $\beta$ (IL-1 $\beta$ ). In addition, activated microglia release matrix metalloproteinases (MMPs) to decompose the extracellular matrix, and then migrate to the infarction area to clear dead cells and blood clots, which causes serious blood-brain barrier (BBB) damage. This further induces the recruitment and migration of circulatory neutrophils, monocytes and macrophages into the ischemic brain (3).

MMPs in the brain are primarily released from microglia/macrophages, neutrophils, endothelial cells and neuronal cells (4), and are divided into five classes based on in vitro substrate analysis, including collagenases (MMP-1, 8, 13 and 18), gelatinases (MMP-2 and -9), stromelysins (MMP-3, 7, 10 and 11), membrane-type MMPs (MMP-14, -15, -16 and -21) and matrilysins (5). Following ischemic stroke, MMP-9 activity markedly rises in association with BBB breakdown, which is significantly attenuated following MMP inhibition or MMP-9 gene depletion $(6,7)$. Therefore, activation of MMP-9 may serve a crucial role in the pathogenesis of acute and subacute brain damage following ischemic stroke.

Besides MMPs, previous studies have indicated that the transcription factor nuclear factor $(\mathrm{NF})-\kappa \mathrm{B}$ serves as a key regulator of cell survival and inflammation (8). NF- $\kappa$ B typically holds in the cytoplasm as an inactivated form by the inhibitor protein $\mathrm{I} \kappa \mathrm{B}(9,10)$. Following ischemic stroke, I $\mathrm{B}$ 
phosphorylates and is degraded by proteasomes. Subsequently, $\mathrm{NF}-\kappa \mathrm{B}$ may translocate from the cytoplasm into nuclei and attach to relevant DNA binding sites on the promoter region of genes. Downstream inflammatory signals including IL-1 $\beta$, IL-6, inducible nitric oxide synthase (iNOS) and MMPs subsequently initiate transcription and protein expression $(11,12)$, promoting the inflammatory response.

3-(5-hydroxymethyl-2-furyl)-1-benzyl-indazole (YC-1), a chemically synthetic benzylindazole compound, has been reported to inhibit LPS-induced production of NO, prostaglandin E2, iNOS and cyclooxygenase-2, and proinflammatory cytokines including TNF- $\alpha$ and IL-1 $\beta$ (13). Therefore, YC-1 has a high potential to be developed as an anti-inflammatory neuroprotective agent. Additionally, YC-1 may downregulate MMP-9 protein expression levels in the human retina, and protect $\mathrm{BBB}$ permeability via inhibiting hypoxia inducible factor 1 accumulation and vascular endothelial growth factor production following ischemia/reperfusion-induced injury in rats $(14,15)$. Previous studies have demonstrated that treatment with 1 or $2 \mathrm{mg} / \mathrm{kg}$ YC-1 does not inhibit the formation of infarction and brain edema, but increases mortality and infarct volume in rats following stroke $(14,16,17)$. In addition Chen et al (18) previously demonstrated that animals treated with $1 \mathrm{mg} / \mathrm{kg}$ YC-1 exhibited reduced infarct volume, but it failed to reduce hemorrhage volume following ischemia (18). These results indicated that an effective dose of YC-1 for neuroprotection following ischemic stroke requires further assessment.

The present study investigated the neuroprotective effect of YC-1 in a dose-dependent manner in mice subjected to transient focal cerebral ischemia. Whether administration of YC-1 may reduce $\mathrm{NF}-\kappa \mathrm{B}$-driven inflammatory responses in cultured neurons exposed to oxygen and glucose deprivation condition (OGD), and in mice following ischemic stroke, was assessed.

\section{Materials and methods}

Primary cortical neuron culture. All animal experiments were approved by the Institutional Animal Care and Use Committee of National Cheng Kung University (Tainan, Taiwan). Cultured neurons were obtained from cerebral cortices of 2 day-old Sprague-Dawley rats $(n=48$; purchased from the Research Animal Care of National Cheng Kung University Medical Center) according to methods described previously $(19,20)$. Rats were housed at $24 \pm 1^{\circ} \mathrm{C}, 60 \%$ humidity and on a $12 \mathrm{~h}$ light/dark cycle. The neonatal rats received nutrition from their mother. Following sacrifice, the cortices were separated in ice-cold Dulbecco's modified Eagle's medium (DMEM; Gibco; Thermo Fisher Scientific, Inc., Waltham, MA, USA). Tissue chunks were crushed and incubated with Hank's Balanced Salt Solution (HBSS; Gibco; Thermo Fisher Scientific, Inc.) containing papain (Sigma-Aldrich; Merck KGaA, Darmstadt, Germany) and DNase I (Bionovas Biotechnology Co., Ltd., Toronto, ON, Canada) at $37^{\circ} \mathrm{C}$ to dissociate the cells. After 30 min incubation, $10 \%$ heat-inactivated fetal bovine serum (FBS; Gibco; Thermo Fisher Scientific, Inc.) was added to cell suspensions to terminate the reaction. Cells were then were centrifuged at $4^{\circ} \mathrm{C}, 800 \mathrm{x} \mathrm{g}$ for $10 \mathrm{~min}$. The pellets were collected and suspended in DMEM with $10 \% \mathrm{FBS}$, and then were filtrated using a $70 \mu \mathrm{m}$ cell strainer (BD Biosciences,
Franklin Lakes, NJ, USA). Dissociated cells were plated onto poly-D-lysine-coated petri dishes and incubated at $37^{\circ} \mathrm{C}$ in a humidified incubator with $5 \% \mathrm{CO}_{2}$. A total of $4 \mathrm{~h}$ after plating, the culture medium was replaced by neurobasal medium containing $25 \mu \mathrm{M}$ glutamate, $0.5 \mathrm{mM}$ L-glutamine, and $2 \%$ B27 supplement (Invitrogen; Thermo Fisher Scientific, Inc.). The culture medium was replaced every 3 days. Cultured neurons were allowed to grow for 7-14 days.

$O G D$. The model of stroke in primary cortical neuronal culture cells were achieved by combining hypoxia with aglycemia according to a method described previously (19). The OGD medium consisted of HBSS that was lacking glucose and that had previously been infused with $\mathrm{N}_{2}$ for $30 \mathrm{~min}$. The cultured neurons were pretreated with dimethyl sulfoxide (0.1\% DMSO; Sigma-Aldrich; Merck KGaA) as a vehicle, or $30 \mathrm{mM} \mathrm{YC-1}$ for $30 \mathrm{~min}$, and then were incubated with OGD medium and transferred to an anaerobic chamber at $37^{\circ} \mathrm{C}$ in an $\mathrm{N}_{2}$-enriched atmosphere for $2 \mathrm{~h}$. Following the deprivation period, cultured neurons were incubated in neurobasal medium under normal conditions (a humidified incubator with $5 \% \mathrm{CO}_{2}$ at $37^{\circ} \mathrm{C}$ ).

Electrophoretic mobility shift assay (EMSA). EMSA was performed according to a method described previously (21). Nuclear proteins were extracted using an NE-PER Nuclear Protein Extraction kit (Thermo Fisher Scientific, Inc.) according to the manufacturer's protocol. An EMSA assay was carried out using a digoxigenin (DIG) gel shift kit (Roche Applied Science, Penzberg, Germany). DIG was labeled with $3.85 \mathrm{pmol} / \mu 1 \mathrm{NF}-\kappa \mathrm{B}$ Gel Shift Oligonucleotides with the following primer sequences: Forward, 5'-AGTTGAGGC GACTTTCCCAGGC-3' and reverse, 3'-TCAACTCCGCTG AAAGGGTCCG-5' (Santa Cruz Biotechnology, Inc., Dallas, TX, USA). Nuclear protein $(10 \mu \mathrm{g})$ was incubated with a DIG-labeled probe for $30 \mathrm{~min}$ on ice, electrophoresed on a $6 \%$ polyacrylamide gel and subsequently transferred onto a hybridization transfer membrane (PerkinElmer, Inc., Waltham, MA, USA). The DIG-labeled probe was recognized by an alkaline phosphatase-conjugated anti-DIG antibody (1:5,000; cat. no. 03353591910; DIG gel shift kit, Roche Applied Science) at room temperature for $30 \mathrm{~min}$ and was detected by a luminescent image analyzer (Fujifilm LAS-3000; Fuji Photo Film Co., Tokyo, Japan).

Gelatin zymography. Cultured neurons (at days 7-14) were pretreated with vehicle (DMSO) or YC-1 (30 $\mu \mathrm{M})$ for $30 \mathrm{~min}$, and then were exposed to OGD for $2 \mathrm{~h}$. The supernatants were collected $48 \mathrm{~h}$ post-treatment and were stocked at $-20^{\circ} \mathrm{C}$ until required. Samples $(10 \mu \mathrm{l})$ were mixed with sample buffer and loaded onto a $7.5 \%$ SDS-PAGE gel containing $0.125 \%$ gelatin (Sigma-Aldrich; Merck KGaA). Following electrophoresis, the gel was washed with $2.5 \%$ Triton X-100 buffer (J.T. Baker, PA, USA) three times. The gel was incubated in $100 \mathrm{ml} 50 \mathrm{mM}$ Tris- $\mathrm{HCl}$ (pH 7.4) containing $5 \mathrm{mM} \mathrm{CaCl}_{2}, 200 \mathrm{mM} \mathrm{NaCl}$, and $0.2 \%$ Brij 35 (Sigma-Aldrich; Merck $\mathrm{KGaA}$ ) at $37^{\circ} \mathrm{C}$ overnight. The gel was stained with $0.25 \%$ Coomassie blue R-250 (Sigma-Aldrich; Merck KGaA) at room temperature for $60 \mathrm{~min}$.

Western blotting. Nuclear and cytoplasmic proteins were extracted by using the NE-PER Nuclear Protein Extraction 
kit (Thermo Fisher Scientific, Inc.) according to the manufacturer's protocol. The protein concentration of each sample was determined with a Bicinchoninic Acid protein assay kit (Thermo Fisher Scientific, Inc.). Sample proteins (20-50 $\mu \mathrm{g}$ ) were separated by $10 \%$ SDS-PAGE and subsequently transferred onto polyvinylidene difluoride membranes. Membranes were blocked with 5\% skimmed milk in phosphate buffered saline with $0.5 \%$ Tween-20 (PBST) buffer for $30 \mathrm{~min}$ at room temperature, and then were incubated with primary antibodies against phosphorylated (p)-IкB- $\alpha$ (cat. no. sc-8404; 1:500; Santa Cruz Biotechnology, Inc., Dallas, TX, USA) and NF-кB (ADI-KAS-TF110-D; 1:500; Enzo Life Sciences, Inc., Farmingdale, NY, USA) at $4^{\circ} \mathrm{C}$ overnight. After washing, the membranes were incubated with a horseradish peroxidase (HRP)-conjugated secondary antibody (cat. no. AP192P; anti-mouse; cat. no. AP307P; anti-rabbit; 1:2,000; Merck KGaA) for $1 \mathrm{~h}$ at room temperature, and then washed with PBST. Proteins were visualized with an Enhanced Chemiluminescence kit (GE Healthcare Bio-Sciences, Pittsburgh, PA, USA). Membranes were then probed for GAPDH (cat. no. GTX100118; 1:500; GeneTex, Inc., Irvine, CA, USA) or transcription factor II D (cat. no. sc-204; 1:200; Santa Cruz Biotechnology, Inc.) as an internal control. Optical densities were measured using Multi Gauge V3.0 (Fuji Photo Film Co.) on a Luminescent Image Analyzer (Fujifilm LAS-3000; Fuji Photo Film Co.).

Animal preparation, experimental model and drug administration. Adult male C57 black (C57BL/B6) mice, (age, 8 weeks; n=34; weight, 20-26 g; obtained from Research Animal Care of National Cheng Kung University Medical Center), were housed at an ambient temperature of $\sim 24 \pm 1^{\circ} \mathrm{C}$ and were allowed free access to food and water prior to and following surgery. For the surgical procedures on mice, 1-2\% halothane (Sigma-Aldrich; Merck $\mathrm{KGaA}$ ) in $\mathrm{N}_{2} \mathrm{O}: \mathrm{O}_{2}(70: 30 \%)$ was used for anesthesia. The study was approved by the Research Animal Care of National Cheng Kung University Medical Center.

Focal cerebral ischemia was employed by intra-arterial suture occlusion of the proximal right middle cerebral artery (MCA) according to a method described previously (22). Briefly, the external carotid artery (ECA), internal carotid artery (ICA) and the pterygopalatine artery of the ICA were exposed under an operating microscope. A silicone rubber coated nylon suture was inserted into the ICA via a slit in the ECA. The suture was advanced 7.5-8.5 mm along the ICA until the tip occluded the origin of the MCA. Reperfusion was produced by gently removing the suture and closing the incision after $60 \mathrm{~min}$ of MCA occlusion (MCAO). Following surgery, the animals were kept in a cage with a heating lamp, monitored for $2 \mathrm{~h}$ and then transferred into the home cage.

YC-1 was dissolved in a mixture of PEG 400 (30\% PEG 400 in saline). Animals were intravenously administered with either 5 or $25 \mathrm{mg} / \mathrm{kg}$ YC-1, or vehicle (PEG 400) $60 \mathrm{~min}$ before surgery. The zymographic and in situ zymographic were examined after $24 \mathrm{~h}$ following ischemia reperfusion and brain infarction. Immunofluorescence and immunohistochemistry were performed $48 \mathrm{~h}$ after ischemia reperfusion.

Neurobehavioral testing, locomotor impairment tests and body weight measurements. All assessment was based on a method described previously $(22,23)$. Body weight was measured daily. A variety of sensory-motor tests were conducted $48 \mathrm{~h}$ after ischemia-reperfusion. Two neurological grading systems were used: A sensorimotor grading scale and a grading scale of $0-28$. The score given to each mouse at the completion of testing was the summation of seven individual test scores (body symmetry, gait, climbing of 45 angle plate, circling behavior, front limb symmetry, compulsory circling and whisker response). Locomotor impairment was assessed using a rota-rod treadmill (model 47700; Ugo Basile Biological Research Apparatus, Varese, Italy). Latency to fall off the rota rod was determined $48 \mathrm{~h}$ after stroke. Mice were conditioned to a rota-rod initially with a fixed speed that was set at $5 \mathrm{rpm}$ for $1 \mathrm{~min}$. A total of $10 \mathrm{~min}$ after the completion of the fixation rota-rod test, the speed was accelerated from 2 to $10 \mathrm{rpm}$ over a period of $5 \mathrm{~min}$. Each test was repeated three times, and the time spent on the rod was recorded. Mice that remained on the rota-rod after 5 min were given a maximum score of $300 \mathrm{sec}$.

Animal sacrifice and quantification of ischemic brain damage. All assessment was based on a method described previously (22). After $24(n=14)$ or $48 \mathrm{~h}(\mathrm{n}=20)$, mice were sacrificed under anesthesia (4\% chloral hydrate/1 ml/100 g; Sigma-Aldrich; Merck KGaA) and perfused transcardially with $4 \%$ formaldehyde in $0.1 \mathrm{M}$ PBS following ischemia-reperfusion. After postfixation and dehydration, the brains were embedded in Optimal Cutting Temperature compound (Tissue-Tek; Miles Laboratories Inc., Elkhart, IN, USA) and frozen in liquid nitrogen. The brains were sectioned into 10 - or $40 \mu \mathrm{m}$ pieces at eight preselected coronal levels on a cryostat (HM-500O; Microm International GmbH, Walldorf, Germany). Sections were mounted on poly-l-lysine-coated (Sigma-Aldrich; Merck $\mathrm{KGaA}$ ) slides and dried at $37^{\circ} \mathrm{C}$ overnight, and then were stored at $-20^{\circ} \mathrm{C}$.

Brain sections $(40 \mu \mathrm{m})$ were stained with $0.5 \%$ cresyl violet for $4 \mathrm{~h}$ at room temperature. Under a light microscope, the areas of neuronal perikarya displaying typical morphologic features of ischemic damage were delineated using a computerized image analyzer (MCID Elite; Imaging Research Inc., St. Catharines, ON, Canada). Infarct volumes were measured and expressed as a percentage of the contralateral hemisphere volume (the contralateral hemisphere area minus the ipsilateral non-ischemic hemisphere area, and then divided by the contralateral hemisphere area). The ipsilateral brain edema was expressed as a percentage index relative to the volume of the left hemisphere (ischemic hemisphere area divide by contralateral hemisphere area minus infarct volume).

Immunofluorescence staining. Immunofluorescence staining was performed based on a method described previously (24). A total of $48 \mathrm{~h}$ following ischemic insult, brain sections were post-fixed in $4 \%$ paraformaldehyde in PBS for $5 \mathrm{~min}$ at room temperature and incubated with alcohol:acetic acid at a ratio of 2:1 for $30 \mathrm{~min}$. Following washing, the brain sections were incubated with polyclonal mouse anti-p-IкB (cat. no. sc-8404;

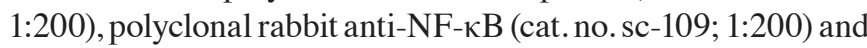
polyclonal goat anti-MMP-9 (cat. no. sc-6841; 1:200) primary antibodies, all purchased from Santa Cruz Biotechnology, 


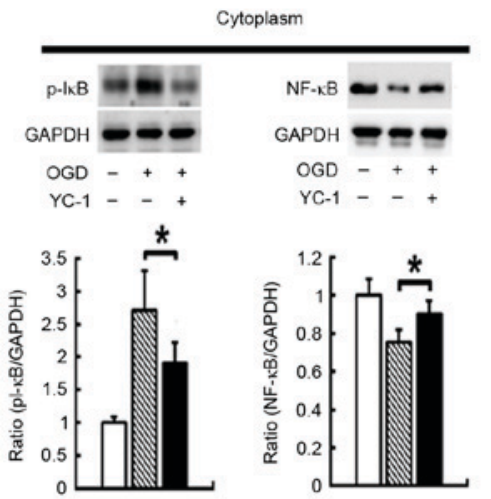

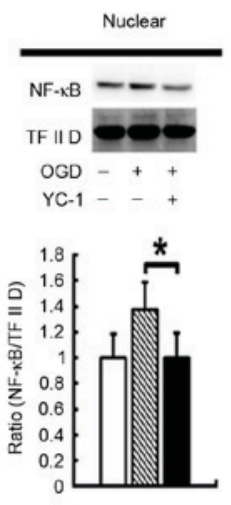

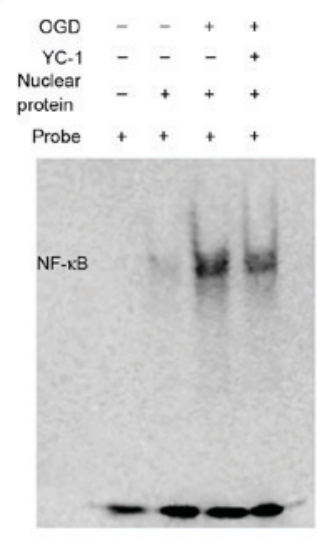

NF-KB DNA binding activity

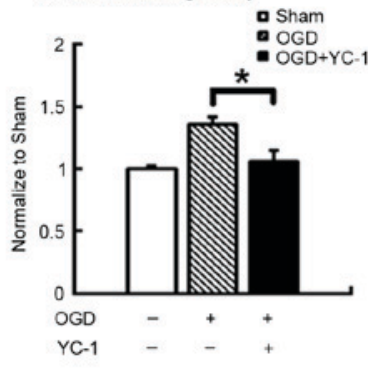

p-lkB
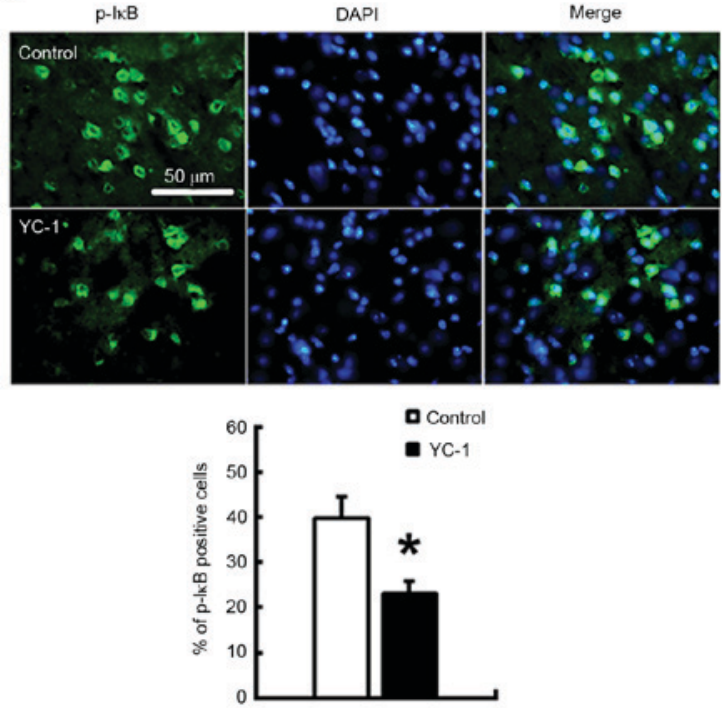

NF-KB

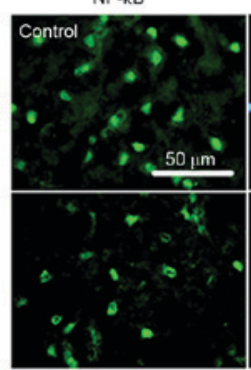

DAPI
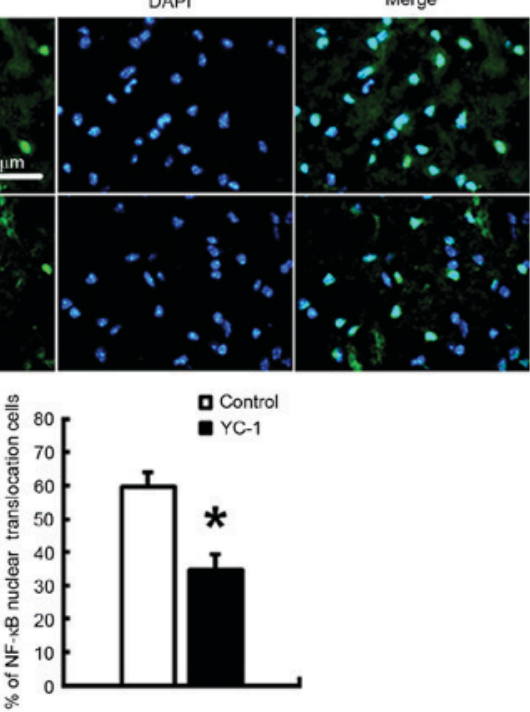

Figure 1. YC-1 decreases NF- $\mathrm{BB}$ binding activity and translocation in vitro and in vivo. Primary cortical neuronal cultures were exposed to OGD. (A) Representative western blot images and quantification demonstrating that $30 \mu \mathrm{M} Y \mathrm{Y}-1$ treatment decreased the phosphorylation of the NF- $\kappa \mathrm{B}$ inhibitory protein $\mathrm{p}-\mathrm{I} \kappa \mathrm{B}$, and inhibited $\mathrm{NF}-\kappa \mathrm{B}$ translocation from the cytoplasm to nucleus. GAPDH and TF II D served as internal controls for $\mathrm{p}-\mathrm{I} \kappa \mathrm{B}$ and $\mathrm{NF}-\kappa \mathrm{B}$, respectively. (B) YC-1 significantly reduced the increased NF- $\kappa$ B binding activity. In the ischemic brain, $25 \mathrm{mg} / \mathrm{kg} \mathrm{YC}-1 \mathrm{significantly} \mathrm{reduced} \mathrm{the} \mathrm{density} \mathrm{of}(\mathrm{C}) \mathrm{pI}-\kappa \mathrm{B}$ and (D) NF- $\kappa$ B translocation, as assessed by immunofluorescence. Data are expressed as the mean \pm standard deviation ( $\mathrm{n}=5 / \mathrm{group}$ ). ${ }^{*} \mathrm{P}<0.05 \mathrm{vs.}$ control. YC-1, 3-(5-hydroxymethyl-2-furyl)-1-benzyl-indazole; OGD, oxygen and glucose deprivation; NF- $\mathrm{B}$, nuclear factor- $\kappa \mathrm{B}$; TF II D, transcription factor II D.

Inc., at $4^{\circ} \mathrm{C}$ overnight. Following this, the brain sections were incubated with corresponding fluorescein isothiocyanate-conjugated IgG secondary antibodies [cat. no. AP124F (anti-mouse IgG), 1:2,000; Chemicon International lnc., MA, USA; 111-095-003 (anti-rabbit IgG), 305-095-003 (anti-goat IgG), 1:5,000; Jackson ImmunoResearch Laboratories Inc., West Grove, PA, USA], for $1 \mathrm{~h}$ at room temperature. Sections were mounted with Dapi Fluoromount- $G^{\circledR}$ (SouthernBiotech, Birmingham, AL, USA) for $10 \mathrm{sec}$ at room temperature. A Zeiss Axioskop 2 Mot microscope equipped with a digital CoolSnap-Pro cf camera (Media Cybernetics, Inc., Rockville, MD, USA) was used to image brain sections.

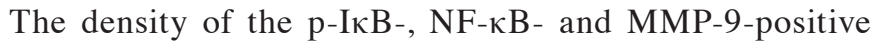
cells were calculated relative to the total number of DAPI-stained cells.

Immunohistochemistry. Immunohistochemistry staining was performed based on a method described previously (21).
A total of $48 \mathrm{~h}$ following ischemic insult, brain sections were post-fixed in $4 \%$ paraformaldehyde in PBS for $5 \mathrm{~min}$ at room temperature and subsequently incubated with alcohol:acetic acid at a ratio of $2: 1$ for $30 \mathrm{~min}$. Following washing, the brain sections were incubated at $4^{\circ} \mathrm{C}$ overnight with primary antibodies against mouse anti-rat monoclonal ED-1 (cat. no. 120405, 1:200; Serotec, Raleigh, NC, USA), anti-goat myeloperoxidase (cat. no. sc-16129, 1:200; Santa Cruz Biotechnology, Inc.) and anti-rabbit iNOS (cat. no. AB5382, 1:200; EMD Millipore, Billerica, MA, USA), and were subsequently developed using a DAB Peroxidase (HRP) Substrate kit (Sigma-Aldrich; Merck KGaA). Sections were co-incubated with hematoxylin for $3 \mathrm{~min}$ at room temperature. The protein expression of brain sections were measure using a Zeiss Axioskop 2 Mot microscope equipped with a digital CoolSnap-Pro cf camera and a semi-automated image analysis system (MCID Elite; Imaging Research Inc.). 
A
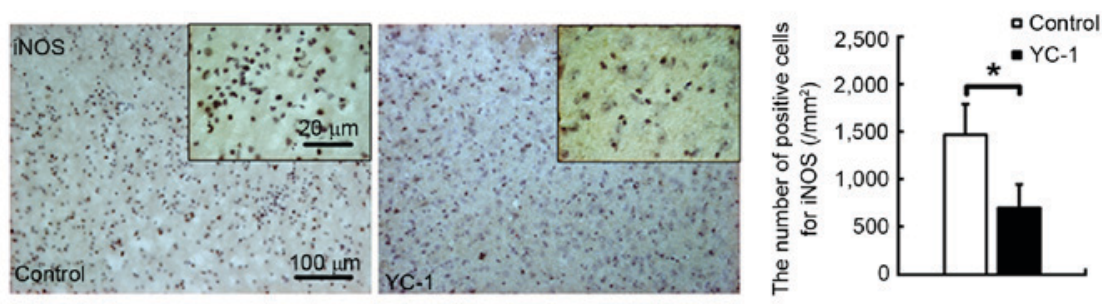

B
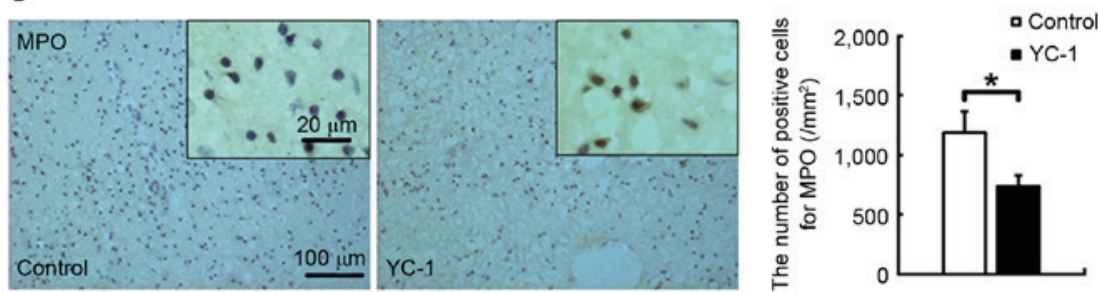

C
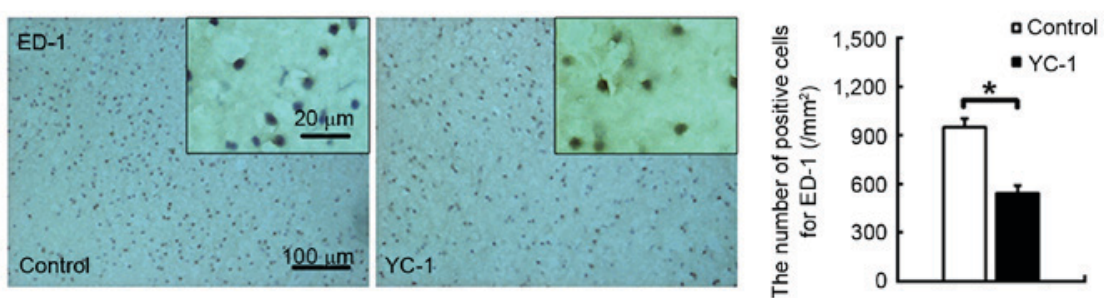

Figure 2. YC-1 reduces inflammatory cellular infiltration in the ischemic brain. Relative to controls, $25 \mathrm{mg} / \mathrm{kg}$ YC-1 treated animals had reduced numbers of (A) iNOS, (B) MPO and (C) ED-1-immunopositive cells at $48 \mathrm{~h}$ post-insult. Scale bar=100 $\mu \mathrm{m}$. Data are expressed as the mean \pm standard deviation (n=5/group). " $\mathrm{P}<0.05$ vs. control. YC-1, 3-(5-hydroxymethyl-2-furyl)-1-benzyl-indazole; iNOS, inducible nitric oxide synthase; MPO, myeloperoxidase.

Measurement of $B B B$ disruption. The integrity of the $\mathrm{BBB}$ was investigated with Evans blue dye extravasation according to previous reports with a few modifications (14). Animals were intravenously administered with either $25 \mathrm{mg} / \mathrm{kg} \mathrm{YC}-1$ $(n=7)$, or vehicle (PEG 400, n=7) $60 \mathrm{~min}$ prior to surgery. Following surgery, $0.5 \mathrm{ml} 4 \%$ Evans blue dye (Sigma-Aldrich; Merck KGaA) solution in saline was administered intravenously. A total of $24 \mathrm{~h}$ after injury, animals were anesthetized and then sacrificed by transcardial perfusion with ice-cold PBS. The brain was dissected out and homogenized in 50\% trichloro acetic acid (Sigma-Aldrich; Merck KGaA) solution. After homogenization, samples were centrifuged at $4^{\circ} \mathrm{C}$, $20,000 \mathrm{x}$ g for $10 \mathrm{~min}$, the supernatants were collected and diluted with ethanol (1:3). The fluorescence was quantified at an excitation wavelength of $620 \mathrm{~nm}$ and an emission wavelength of $680 \mathrm{~nm}$ by a Fluoroskan Ascent ${ }^{\mathrm{TM}}$ FL Microplate Fluorometer (Thermo Fisher Scientific, Inc.). Dye in samples was determined as $\mu \mathrm{g} / \mathrm{g}$ tissue from a standard curve plotted using known amounts of dye.

Statistical analysis. Data were analyzed using SPSS 17.0 (SPSS Inc., Chicago, IL, USA). Data are expressed as the mean \pm standard deviation. Unpaired Student's t-test or one-way analysis of variance followed by least significant difference post hoc comparison was used to evaluate differences between groups. Neurobehavioral scores were expressed as the median $\pm 95 \%$ confidence interval and were analyzed by Mann-Whitney $\mathrm{U}$ test. ${ }^{*} \mathrm{P}<0.05$ was considered to indicate a statistically significant difference.

\section{Results}

$Y C-1$ decreases $N F-\kappa B$ binding activity and translocation in vitro and in vivo. In primary cortical neuronal cultures exposed to OGD, groups pretreated for 30 min with $30 \mu \mathrm{M}$ YC-1, compared with controls, exhibited effectively reduced phosphorylation of the $\mathrm{NF}-\kappa \mathrm{B}$ inhibitory protein $\mathrm{p}-\mathrm{I} \kappa \mathrm{B}$ in the cytoplasm. Additionally, YC-1 pretreatment significantly restored decreased $\mathrm{NF}-\kappa \mathrm{B}$ protein expression levels in cytoplasm, and attenuated the amount of $\mathrm{NF}-\kappa \mathrm{B}$ in the nucleus (Fig. $1 \mathrm{~A} ; \mathrm{P}<0.05$ ). The specific $\mathrm{NF}-\kappa \mathrm{B}$ binding activity was assessed by EMSA. YC-1 pretreatment significantly reduced upregulation of specific $\mathrm{NF}-\kappa \mathrm{B}$ binding activity (Fig. 1B; P<0.05). Additionally, C57BL/B6 mice were pretreated with a vehicle or $25 \mathrm{mg} / \mathrm{kg}$ YC-1 by intravenous injection $60 \mathrm{~min}$ before induction of transient focal cerebral ischemia. YC-1 treatment significantly reduced the

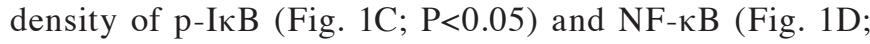
$\mathrm{P}<0.05)$ translocation in the area of brain injury at $48 \mathrm{~h}$ post-insult.

$Y C-1$ reduces inflammatory cellular infiltration in the ischemic brain. Adult male C57BL/B6 mice were subjected to transient focal cerebral ischemia. In the ischemic brain at $48 \mathrm{~h}$ post-insult, the $25 \mathrm{mg} / \mathrm{kg}$ YC-1-treated group exhibited a $52.5 \%$ reduction of iNOS-positive cells (Fig. 2A; $\mathrm{P}<0.05$ ). Additionally, the YC-1 treated group exhibited significantly reduced numbers of neutrophils (Fig. $2 \mathrm{~B} ; \mathrm{P}<0.05$ ) and activated microglia/macrophages (Fig. $2 \mathrm{C} ; \mathrm{P}<0.05$ ) by 37.1 and $36.5 \%$, 
as assessed by the density of myeloperoxidase (MPO) and ED-1 positive cells, respectively.

YC-1 reduces MMP-9 expression and activity in vitro and in vivo. In primary cortical neuronal cultures exposed to OGD, the vehicle-treated group demonstrated increased MMP-9 enzyme activation, compared with the control group. However, pretreatment with YC-1 significantly attenuated this activation, as assessed by gelatin-dependent zymography (Fig. 3A; $\mathrm{P}<0.05)$. However, no significant differences were observed in MMP-2 enzyme activation. Furthermore, in the ischemic brain at $48 \mathrm{~h}$ post-insult, $\mathrm{YC}-1$ pretreatment significantly reduced the density of MMP-9-positive cells (Fig. 3B; P<0.05).

YC-1 markedly ameliorates BBB disruption in mice following ischemic stroke. Evans blue dye was used as a marker to evaluate the effect of YC-1 on BBB permeability. Evans blue leakage increased significantly in the brains of control group mice subjected to MCAO and $24 \mathrm{~h}$ reperfusion, whereas administration of $25 \mathrm{mg} / \mathrm{kg}$ YC-1 to mice markedly ameliorated the Evans blue leakage (Fig. 4A; $\mathrm{P}<0.05$ ).

YC-1 reduces ischemic brain infarction and edema in mice subjected to transient focal cerebral ischemia. Adult mice were subjected to transient focal cerebral ischemia. Compared with controls, 5 and $25 \mathrm{mg} / \mathrm{kg}$ YC-1 pretreated mice had infarction volumes reduced by 27.5 and $57.5 \%$, respectively, and individual cortical lesion sizes reduced by $68.1 \%$ in the $25 \mathrm{mg} / \mathrm{kg}$ YC-1-treated group at $48 \mathrm{~h}$ post-insult. Furthermore, $25 \mathrm{mg} / \mathrm{kg}$ YC-1-treated mice demonstrated significantly reduced brain edema at $48 \mathrm{~h}$ after reperfusion (Fig. 4B; $\mathrm{P}<0.05$ ).

YC-1 improves neurological behavioral scores and rota-rod motor performance tests following cerebral ischemia-reperfusion. Administration of 5 and $25 \mathrm{mg} / \mathrm{kg}$ YC-1 to mice by intravenous injection resulted in significantly improved sensory, motor and 28-point neurologic scores taken $48 \mathrm{~h}$ post-insult compared with the control group. Furthermore, the $25 \mathrm{mg} / \mathrm{kg}$ YC-1-treated group demonstrated a longer running time in the rota-rod motor performance test (Table I; P<0.05).

\section{Discussion}

The results of the present study demonstrated that YC-1

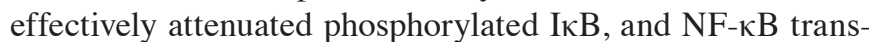
location and binding activity, therefore downregulting iNOS expression following stroke. Additionally, YC-1 effectively attenuated increased infiltration of neutrophils and activated microglia/macrophages following ischemia-reperfusion. YC-1 effectively reduced stroke-induced MMP-9 enzyme activity and MMP-9 protein expression levels in vivo and in vitro. In addition, YC-1-treated mice had significantly reduced BBB disruption and brain infarct volume, and improved neurobehavioral outcomes.

A previous report has indicated that YC-1 inhibits NF-kB activation and induces apoptosis in human prostate cancer cells (25). In addition, YC-1 reduces NF- $\mathrm{KB}$ activation and the release of cytokines, including TNF- $\alpha$, ILs-1 $\beta$, IL- 6 and IL-8 in endotoxemic mouse models $(13,26)$. Previous reports
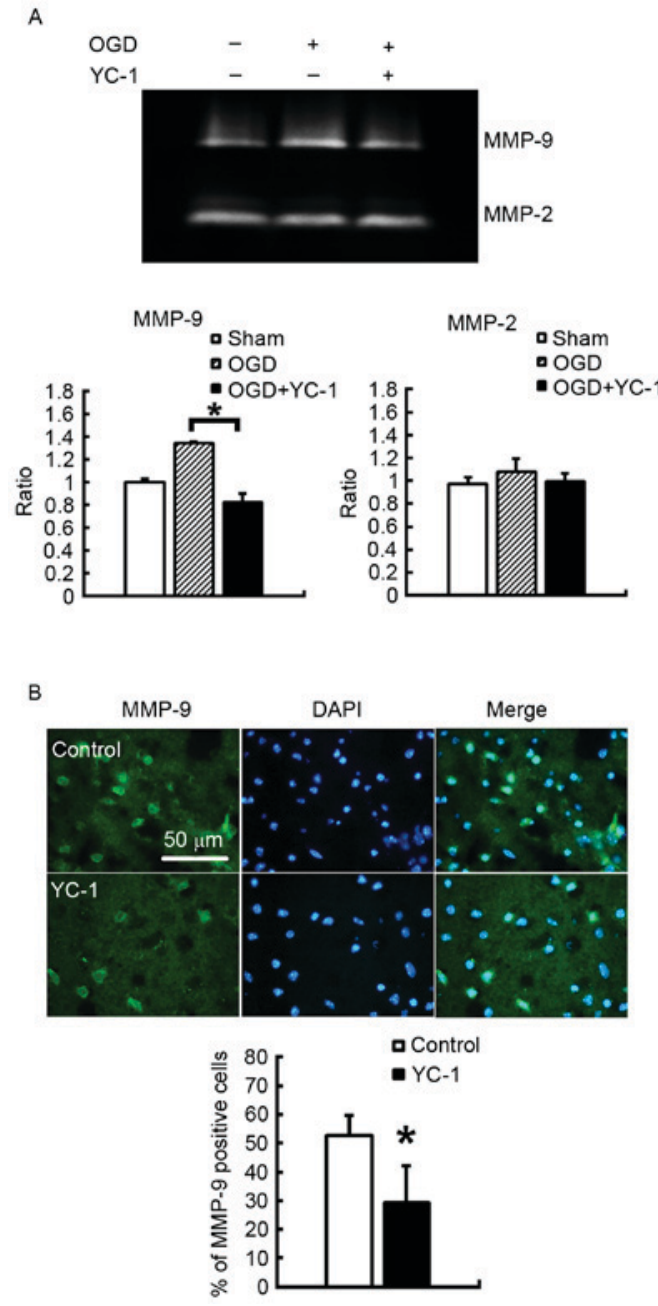

Figure 3. YC-1 reduces MMP-9 expression and activity in vitro and in vivo in primary cortical neuronal cultures exposed to OGD. (A) Gelatin-dependent zymography demonstrated that $30 \mu \mathrm{M}$ YC-1 significantly suppressed MMP-9 activity $48 \mathrm{~h}$ after reperfusion. In the controls and the YC-1 treated animals $48 \mathrm{~h}$ after reperfusion ( $\mathrm{n}=6 /$ group). (B) Immunofluorescence staining revealed that $25 \mu \mathrm{M}$ YC-1 significantly suppressed MMP-9 protein expression levels ( $\mathrm{n}=5 /$ group). Data are expressed as the mean \pm standard deviation. ${ }^{*} \mathrm{P}<0.05$ vs. control. YC-1, 3-(5-hydroxymethyl-2-furyl)-1-benzyl-indazole; MMP, matrix metalloproteinase.

have also indicated that YC-1 inhibits iNOS expression via suppression of NF- $\kappa B$ activity $(9,13,26)$. This was further confirmed by YC-1 mediated inhibition on a variety of the NF- $\kappa \mathrm{B}$-driven cytotoxic immune mediators in vitro and in vivo. Consistent with these previous reports, the present study demonstrated that YC-1 effectively attenuated the phosphorylation of $\mathrm{I} \mathrm{KB}$, and NF- $\mathrm{\kappa B}$ translocation and binding activity, thus downregulating iNOS expression levels following ischemic stroke.

Following cerebral ischemia, microglia/macrophages readily become activated and consequently release a variety of cytotoxic immune mediators and proinflammatory cytokines; thus, serve a crucial role in the pathogenesis of delayed brain injury. The present study observed that YC-1 robustly reduced neutrophil infiltration, microglial/macrophage activation and the generation of a variety of cytotoxic immune mediators in mice subjected to cerebral ischemia. Microglia/macrophages are the primary source of MMP-9 in the brain. MMP-9 
Table I. YC-1 improves sensorimotor and rota-rod motor performance tests following cerebral ischemia-reperfusion.

\begin{tabular}{|c|c|c|c|c|c|c|c|}
\hline \multirow[b]{2}{*}{ Variable } & \multirow{2}{*}{\multicolumn{2}{|c|}{ Weight loss (g) }} & \multicolumn{3}{|c|}{ Neurological behavioral score } & \multicolumn{2}{|c|}{ Rota-rod performance } \\
\hline & & & Sensory & Motor & $\begin{array}{c}\text { 28-point } \\
\text { clinical scale }\end{array}$ & $\begin{array}{c}\text { ACC } \\
(2-10 \mathrm{rpm} / 0-5 \mathrm{~min})\end{array}$ & $\begin{array}{c}\text { FIX } \\
(5 \mathrm{rpm} / \mathrm{min})\end{array}$ \\
\hline Control, & $\mathrm{N}=7$ & $4.8 \pm 1.1$ & $3(2.6-3.4)$ & $2(1.6-2.4)$ & $15(13.1-16.9)$ & $105.5 \pm 107.6$ & $122.6 \pm 147.9$ \\
\hline YC-1 (5 mg/kg) & $\mathrm{N}=6$ & $5.8 \pm 0.7$ & $2(1.7-2.3)^{\mathrm{a}}$ & $1(0.6-1.4)^{\mathrm{a}}$ & $8(7.5-8.5)^{\mathrm{a}}$ & - & - \\
\hline YC-1 $(25 \mathrm{mg} / \mathrm{kg})$ & $\mathrm{N}=7$ & $5.3 \pm 1.0$ & $2(1.5-2.5)^{\mathrm{a}}$ & $1(0.7-1.3)^{\mathrm{a}}$ & $8(6.5-9.5)^{\mathrm{a}}$ & $233.5 \pm 82.5^{\mathrm{a}}$ & $276.3 \pm 42.3^{\mathrm{a}}$ \\
\hline
\end{tabular}

Data are presented as the mean \pm standard deviation (weight and rota-road data) or as the median (neurological behavior score). ${ }^{\mathrm{a}} \mathrm{P}<0.05 \mathrm{vs}$. control. ACC, accelerated speed; FIX, fixed speed.

A
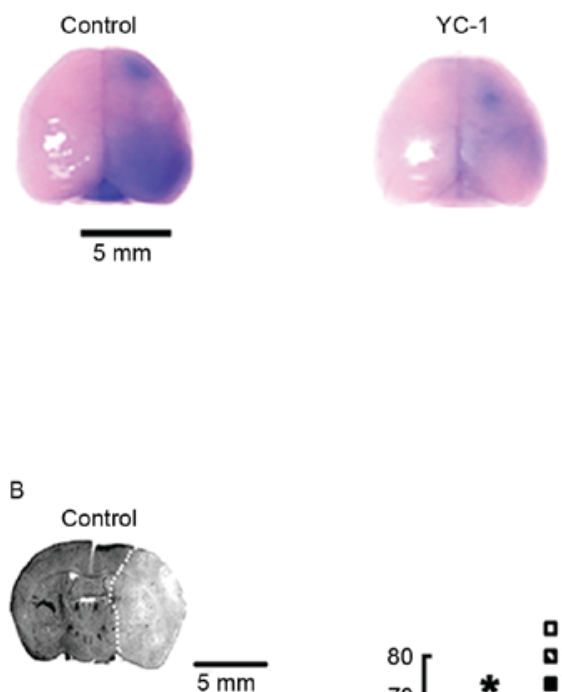

YC-1 $(5 \mathrm{mg} / \mathrm{kg})$

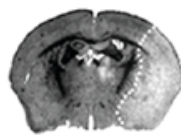

YC-1 $(25 \mathrm{mg} / \mathrm{kg})$

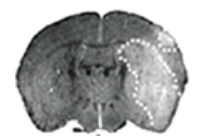

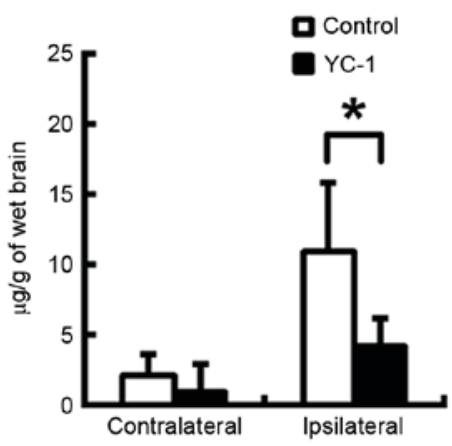
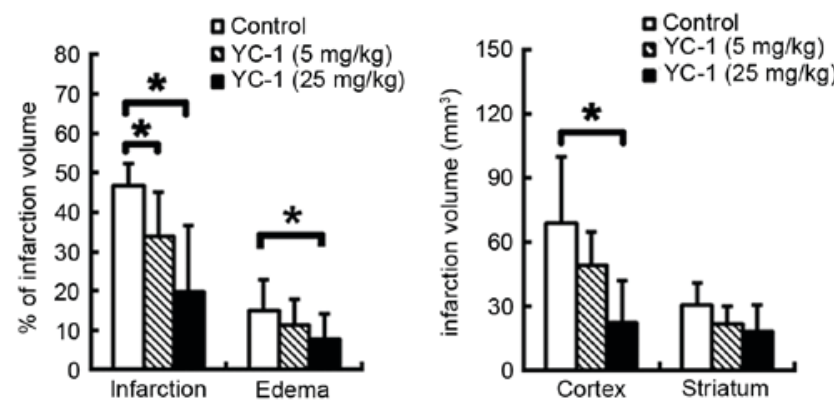

Figure 4. YC-1 markedly ameliorates blood-brain barrier disruption and reduces ischemic brain infarction in mice subjected to MCAO. (A) Representative images of Evans blue extravasation in the whole brain, and quantification of Evans blue leakage in ipsilateral and contralateral hemispheres of MCAO mice following $25 \mathrm{mg} / \mathrm{kg} \mathrm{YC}-1$ (n=7/group). (B) Nissl-stained coronal sections obtained from representative animals which received an intravenous injection of vehicle or YC-1 (5 and $25 \mathrm{mg} / \mathrm{kg}$ ) prior to ischemic onset (control, $\mathrm{n}=7 ; 5 \mathrm{mkg} / \mathrm{kg}$ YC-1, n=6; $25 \mathrm{mg} / \mathrm{kg}$ YC-1, n=7). Scale bar=5 mm. Data are expressed as the mean \pm standard deviation. ${ }^{*}<0.05$ vs. control. MCAO, middle cerebral artery occlusion; YC-1, 3-(5-hydroxymethyl-2-furyl)-1-benzyl-indazole.

activation and overexpression are linked with the process of brain infarction, the formation of hemorrhagic events and brain edema during cerebral ischemic stroke (6). The present study demonstrated that MMP-9 activation and increased expression following stroke was reduced by exogenous YC-1, which was accompanied by amelioration of BBB disruption. This result was consistent with a previous study, in which $2 \mathrm{mg} / \mathrm{kg}$ YC-1 treatment markedly ameliorated ischemia-induced BBB disruption in a rat ischemic stroke model (14), and MMP-9 activation and expression was inhibited by orally administered YC-1 $(1-10 \mathrm{mg} / \mathrm{kg})$ in a balloon injury rat carotid artery model (27).

Previous reports have indicated that 1 or $2 \mathrm{mg} / \mathrm{kg}$ YC-1 treatment does not effective reduce brain infarction volume (14,16-18); however, the results of the present study demonstrated that 5 and $25 \mathrm{mg} / \mathrm{kg}$ YC-1 treatment significantly reduced infarction volumes and improved neurobehavioral outcomes at $48 \mathrm{~h}$ post-insult compared with 


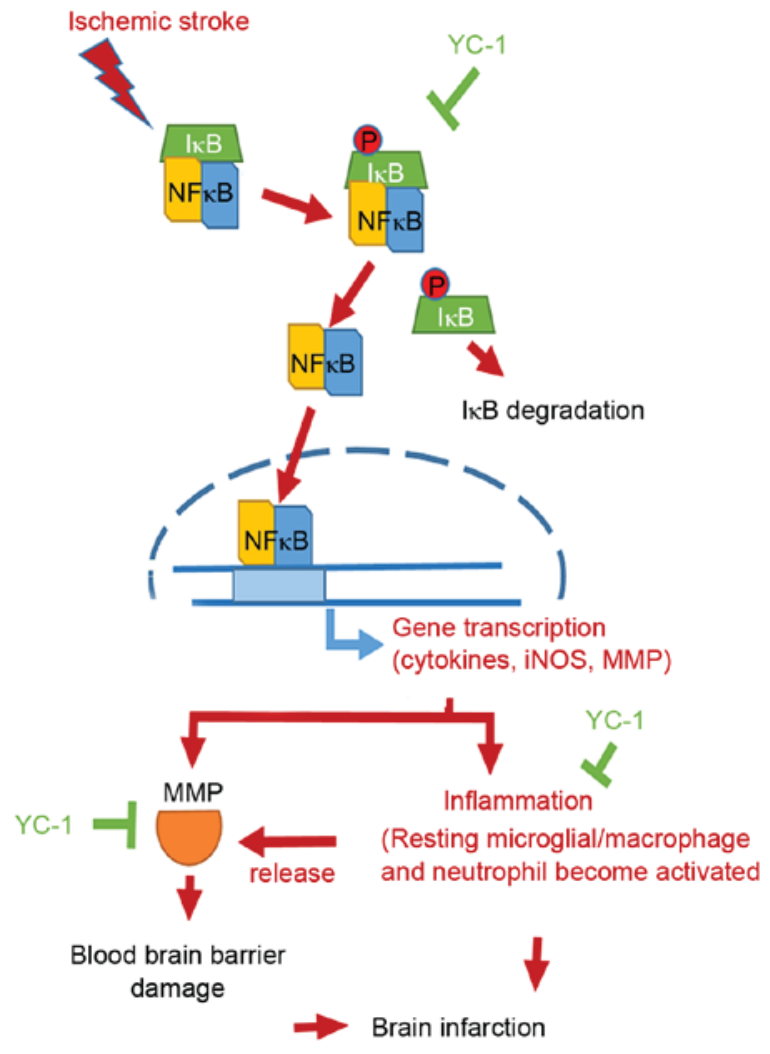

Figure 5. Ischemic stroke schematic diagram. IкB was phosphorylated and then was degraded. Subsequently, NF- $\kappa \mathrm{B}$ translocated from cytoplasm into nuclei and drove downstream inflammatory signals, including iNOS and MMP protein expression. The resting microglia cells became activated and released more MMPs proteins, which caused serious blood-brain barrier damage. Severe inflammatory reactions and MMP overexpression increased brain infarction. Therefore, YC-1 effectively inhibited NF- $\kappa$ B translocation, binding activity and inflammatory cellular infiltration, and inhibited the MMP-9 expression levels following ischemic stroke. YC-1 treatment attenuated brain infarction volume following cerebral ischemia-reperfusion. iNOS, inducible nitric oxide synthase; YC-1, 3-(5-hydroxymethyl-2-furyl)-1-benzyl-indazole; $\mathrm{MMP}$, matrix metalloproteinase; $\mathrm{NF}-\kappa \mathrm{B}$, nuclear factor-Kb.

controls. These results indicated that treatment of $>5 \mathrm{mg} / \mathrm{kg}$ of YC-1 in animals is effective for neuroprotection following ischemic stroke.

From the above results, it may be concluded that YC-1 downregulated iNOS and MMP-9 expression levels by inhibiting the $\mathrm{NF}-\kappa \mathrm{B}$ signaling pathway, reducing inflammatory cellular infiltration, and therefore may ameliorate BBB disruption following ischemic stroke. Via the above mechanism, YC-1 may reduce the brain infarction volume in mice subjected to transient focal cerebral ischemia (Fig. 5).

Yan et al (14) reported no significant differences in the levels of brain edema between $2 \mathrm{mg} / \mathrm{kg}$ YC- 1 and control groups following MCAO and $24 \mathrm{~h}$ reperfusion. In the present study, YC-1 treated animals exhibited dose-responsively attenuated brain edema, and the $25 \mathrm{mg} / \mathrm{kg}$ YC-1 treated group had significantly reduced brain edema $48 \mathrm{~h}$ after reperfusion. A concentration of $>5 \mathrm{mg} / \mathrm{kg} \mathrm{YC}-1$ may reduce brain infarction, but $25 \mathrm{mg} / \mathrm{kg}$ was the optimum concentration for inhibiting brain edema. Inhibited brain edema formation may serve an important role in the neuroprotective mechanism of YC-1.
Fluid accumulation caused by infarcted tissue in the intracellular and extracellular regions of the brain result in brain edema and cell swelling. Brain edema, including cytotoxic and vasogenic edema, was a major cause of morbidity and mortality following ischemic stroke. Cytotoxic edema evolves over minutes to hours and may be reversible, whereas vasogenic edema occurs over hours to days, and is considered an irreversibly damaging process. Cytotoxic edema refers to a cellular swelling resulting from failure of adenosine triphosphate-dependent ion (sodium and calcium) transport. In addition, an alteration in extracellular $\mathrm{pH}$ may affect intercellular $\mathrm{pH}$. This alteration of $\mathrm{pH}$ may affect a variety of ionic channels, including voltage-dependent calcium channels (28). Furthermore, BBB and extracellular matrix disruption, resulting from loss of tight junctions and activation of matrix MMPs, are associated with vasogenic edema formation in ischemic stroke. A previous study indicated that the glial water channel protein aquaporin-4 (AQP4) function serves a critical role in brain water homeostasis following brain injury (29). AQP4 is a member of the aquaporin family of intrinsic membrane proteins that function as water-selective channels in brain. AQP4 is abundantly expressed in astrocytes and serves a key role in the development of brain edema $(30,31)$. Investigation of AQP4-deficient ( $\mathrm{AQP} 4^{-/}$) mice indicated that AQP4 promotes cytotoxic edema but attenuates vasogenic edema following ischemic stroke $(32,33)$. In $\mathrm{AQP} 4^{-/}$mice, acute brain injury following permanent focal cerebral ischemia is attenuated as a result of decreased cytotoxic edema. Future studies should investigate the effects of YC-1 on factors of cytotoxic and vasogenic edema, including ion channels, $\mathrm{pH}$ channels, and AQP40 protein and associated factors of edema following ischemia stroke.

In conclusion, the results of the present study indicated that YC-1 effectively reduces post-stroke inflammatory responses and ameliorates BBB permeability by inhibiting $\mathrm{NF}-\kappa \mathrm{B}$ binding activity and translocation against ischemic stroke. Therefore, YC-1 has high potential to be developed as a treatment for patients following ischemic stroke.

\section{Acknowledgements}

The authors thank Professor Hsin-Yi Hung for her constructive comments on the English language of the manuscript

\section{Funding}

No funding was received.

\section{Availability of data and materials}

The analyzed data sets generated during the study are available from the corresponding author on reasonable request.

\section{Authors' contributions}

WL designed the model, analyzed the data, and wrote the manuscript. ST conceived and planned the experiments. YL contributed to sample preparation. TW and EL conceived, designed the study, and approved the final version of the manuscript. All authors read and approved the final manuscript. 


\section{Ethics approval and consent to participate}

All animal experiments were approved by the Institutional Animal Care and Use Committee of National Cheng Kung University (Tainan, Taiwan).

\section{Consent for publication}

Not applicable.

\section{Competing interests}

The authors declare they have no competing interests.

\section{References}

1. Demaerschalk BM and Yip TR: Economic benefit of increasing utilization of intravenous tissue plasminogen activator for acute ischemic stroke in the United States. Stroke 36: 2500-2503, 2005.

2. Wang Q, Tang XN and Yenari MA: The inflammatory response in stroke. J Neuroimmunol 184: 53-68, 2007.

3. Ceulemans AG, Zgavc T, Kooijman R, Hachimi-Idrissi S, Sarre S and Michotte Y: The dual role of the neuroinflammatory response after ischemic stroke: Modulatory effects of hypothermia. J Neuroinflammation 7: 74, 2010.

4. Savman K, Heyes MP,Svedin P and Karlsson A: Microglia/macrophage-derived inflammatory mediators galectin-3 and quinolinic acid are elevated in cerebrospinal fluid from newborn infants after birth asphyxia. Transl Stroke Res 4: 228-235, 2013.

5. Oikonomidi S, Kostikas K, Tsilioni I, Tanou K, Gourgoulianis KI and Kiropoulos TS: Matrix metalloproteinases in respiratory diseases: From pathogenesis to potential clinical implications. Curr Med Chem 16: 1214-1228, 2009.

6. Hung YC, Chen TY, Lee EJ, Chen WL, Huang SY, Lee WT, Lee MY, Chen HY and Wu TS: Melatonin decreases matrix metalloproteinase-9 activation and expression and attenuates reperfusion-induced hemorrhage following transient focal cerebral ischemia in rats. J Pineal Res 45: 459-467, 2008.

7. Gasche Y,Copin JC, Sugawara T,Fujimura Mand Chan PH: Matrix metalloproteinase inhibition prevents oxidative stress-associated blood-brain barrier disruption after transient focal cerebral ischemia. J Cereb Blood Flow Metab 21: 1393-1400, 2001.

8. Ridder DA and Schwaninger M: NF-kappaB signaling in cerebral ischemia. Neuroscience 158: 995-1006, 2009.

9. Hsiao G, Huang HY, Fong TH, Shen MY, Lin CH, Teng CM and Sheu JR: Inhibitory mechanisms of YC-1 and PMC in the induction of iNOS expression by lipoteichoic acid in RAW 264.7 macrophages. Biochem Pharmacol 67: 1411-1419, 2004.

10. Grimm $S$ and Baeuerle PA: The inducible transcription factor NF-kappa B: Structure-function relationship of its protein subunits. Biochem J 290: 297-308, 1993.

11. Bond M, Chase AJ, Baker AH and Newby AC: Inhibition of transcription factor NF-kappaB reduces matrix metalloproteinase-1, -3 and -9 production by vascular smooth muscle cells. Cardiovasc Res 50: 556-565, 2001.

12. SunBZ, Chen L, WuQ, Wang HL, WeiXB,Xiang YX andZhang XM: Suppression of inflammatory response by flurbiprofen following foca cerebral ischemia involves the NF-kappaB signaling pathway. Int J Clin Exp Med 7: 3087-3095, 2014.

13. Lu DY, Tang CH, Liou HC, Teng CM, Jeng KC, Kuo SC, Lee FY and Fu WM: YC-1 attenuates LPS-induced proinflammatory responses and activation of nuclear factor-kappaB in microglia. Br J Pharmacol 151: 396-405, 2007.

14. Yan J, Zhou B, Taheri S and Shi H: Differential effects of HIF-1 inhibition by $\mathrm{YC}-1$ on the overall outcome and blood-brain barrier damage in a rat model of ischemic stroke. PloS One 6: e27798, 2011.

15. DeNiro M, Alsmadi O and Al-Mohanna F: Modulating the hypoxia-inducible factor signaling pathway as a therapeutic modality to regulate retinal angiogenesis. Exp Eye Res 89: 700-717, 2009

16. Zhang Z, Yan J, Taheri S, Liu KJ and Shi H: Hypoxia-inducible factor 1 contributes to $\mathrm{N}$-acetylcysteine's protection in stroke. Free Radic Biol Med 68: 8-21, 2014.
17. Yuan LB, Dong HL, Zhang HP, Zhao RN, Gong G, Chen XM, Zhang LN and Xiong L: Neuroprotective effect of orexin-A is mediated by an increase of hypoxia-inducible factor-1 activity in rat. Anesthesiology 114: 340-354, 2011.

18. Chen C, Ostrowski RP, Zhou C, Tang J and Zhang JH: Suppression of hypoxia-inducible factor-1alpha and its downstream genes reduces acute hyperglycemia-enhanced hemorrhagic transformation in a rat model of cerebral ischemia. J Neurosci Res 88: 2046-2055, 2010.

19. Tai SH, Hung YC, Lee EJ, Lee AC, Chen TY, Shen CC, Chen HY, Lee MY, Huang SY and Wu TS: Melatonin protects against transient focal cerebral ischemia in both reproductively active and estrogen-deficient female rats: The impact of circulating estrogen on its hormetic dose-response. J Pineal Res 50: 292-303, 2011.

20. Lee WT, Lin MH, Lee EJ, Hung YC, Tai SH, Chen HY, Chen TY and Wu TS: Magnolol reduces glutamate-induced neuronal excitotoxicity and protects against permanent focal cerebral ischemia up to 4 hours. PloS One 7: e39952, 2012.

21. Chen TY, Lin MH, Lee WT, Huang SY, Chen YH, Lee AC, Lin HW and Lee EJ: Nicotinamide inhibits nuclear factor-kappa $\mathrm{B}$ translocation after transient focal cerebral ischemia. Crit Care Med 40: 532-537, 2012.

22. Tai SH, Chen HY, Lee EJ, Chen TY, Lin HW, Hung YC, Huang SY, Chen YH, Lee WT and Wu TS: Melatonin inhibits postischemic matrix metalloproteinase-9 (MMP-9) activation via dual modulation of plasminogen/plasmin system and endogenous MMP inhibitor in mice subjected to transient focal cerebral ischemia. J Pineal Res 49: 332-341, 2010.

23. Chen HY, Hung YC, Chen TY, Huang SY, Wang YH, Lee WT, Wu TS and Lee EJ: Melatonin improves presynaptic protein, SNAP-25, expression and dendritic spine density and enhances functional and electrophysiological recovery following transient focal cerebral ischemia in rats. J Pineal Res 47: 260-270, 2009.

24. Chang CC, Tien CH, Lee EJ, Juan WS, Chen YH, Hung YC, Chen TY, Chen HY and Wu TS: Melatonin inhibits matrix metalloproteinase-9 (MMP-9) activation in the lipopolysaccharide (LPS)-stimulated RAW 264.7 and BV2 cells and a mouse model of meningitis. J Pineal Res 53: 188-197, 2012.

25. Huang YT, Pan SL, Guh JH, Chang YL, Lee FY, Kuo SC and Teng CM: YC-1 suppresses constitutive nuclear factor-kappaB activation and induces apoptosis in human prostate cancer cells. Mol Cancer Ther 4: 1628-1635, 2005.

26. Pan SL, Guh JH, Peng CY, Chang YL, Cheng FC, Chang JH, Kuo SC, Lee FY and Teng CM: A potential role of YC-1 on the inhibition of cytokine release in peripheral blood mononuclear leukocytes and endotoxemic mouse models. Thromb Haemost 93: 940-948, 2005.

27. Liu YN, Pan SL, Peng CY, Guh JH, Huang DM, Chang YL, Lin $\mathrm{CH}$, Pai HC, Kuo SC, Lee FY and Teng CM: YC-1 [3-(5'-hydroxymethyl-2'-furyl)-1-benzyl indazole] inhibits neointima formation in balloon-injured rat carotid through suppression of expressions and activities of matrix metalloproteinases 2 and 9. J Pharmacol Exp Ther 316: 35-41, 2006.

28. Zhou W and Jones SW: The effects of external pH on calcium channel currents in bullfrog sympathetic neurons. Biophys J 70: 1326-1334, 1996.

29. Manley GT, Fujimura M, Ma T, Noshita N, Filiz F, Bollen AW, Chan P and Verkman AS: Aquaporin-4 deletion in mice reduces brain edema after acute water intoxication and ischemic stroke. Nat Med 6: 159-163, 2000.

30. Tait MJ, Saadoun S, Bell BA and Papadopoulos MC: Water movements in the brain: Role of aquaporins. Trends Neurosci 31: 37-43, 2008.

31. Rash JE, Yasumura T, Hudson CS, Agre P and Nielsen S: Direct immunogold labeling of aquaporin-4 in square arrays of astrocyte and ependymocyte plasma membranes in rat brain and spinal cord. Proc Natl Acad Sci USA 95: 11981-11986, 1998.

32. Zador Z, Stiver S, Wang V and Manley GT: Role of aquaporin-4 in cerebral edema and stroke. Handb Exp Pharmacol 190: 159-170, 2009.

33. Nielsen S, Nagelhus EA, Amiry-Moghaddam M, Bourque C, Agre P and Ottersen OP: Specialized membrane domains for water transport in glial cells: high-resolution immunogold cytochemistry of aquaporin-4 in rat brain. J Neurosci 17: 171-180, 1997. 